Research Article

\title{
A Multiscale Approach to the Design and Manipulation of Oil-in-Water Emulsion-Based Products
}

\author{
Javier D. Gomez, ${ }^{1,2}$ Diego Pradilla $\mathbb{D}^{2}{ }^{2}$ and Oscar Alvarez $\mathbb{D}^{2}$ \\ ${ }^{1}$ Department of Chemical and Biomolecular Engineering, Vanderbilt University, Nashville, TN, USA \\ ${ }^{2}$ Product and Process Design Group (GDPP), Chemical Engineering Department, Universidad de Los Andes, \\ Carrera 1 No. $18^{a}-10$, Bogotá 111711, Colombia
}

Correspondence should be addressed to Diego Pradilla; d-pradil@uniandes.edu.co

Received 3 August 2020; Revised 11 March 2021; Accepted 29 March 2021; Published 15 April 2021

Academic Editor: Maciej Jaskulski

Copyright (c) 2021 Javier D. Gomez et al. This is an open access article distributed under the Creative Commons Attribution License, which permits unrestricted use, distribution, and reproduction in any medium, provided the original work is properly cited.

\begin{abstract}
Advances in computational technology and high-throughput modeling software have given rise to the tailored design of products that require accurate mathematical relationships for their assessment. Industrial emulsion-based products, ubiquitous to everyday life, are complex systems driven by interfacial phenomena that require quick property-prediction tools for their commercialization. In this work, by means of a multiscale approach, mathematical relationships to model oil-in-water emulsions and that can be applied to any commercial emulsion-based product are proposed. The energy consumption during the emulsification process $\left(E_{v}\right.$, which transitions from monotonic increase to exponential growth at $80 \% \mathrm{w} / w$ ), a parameter responsible for finished product performance, was linked to final product properties at three different levels: (i) molecular, through the dynamics of the interdroplet interactions given their distribution and structure at a microscopic level; (ii) microscopic, through average droplet size yielding an inversely proportional exponential relationship $\left(D_{[4,3]} \propto E_{v}^{-4}\right)$; and (iii) macroscopic, through the plateau value of the elastic modulus and the flow behavior index leading to inversely proportional quadratic relationships $\left(G^{\prime} \propto E_{v}^{-2}\right.$ and $\eta \propto E_{v}^{-2}$, respectively). These relationships are valid at dispersed phase concentrations beyond the $60 \% \mathrm{w} / \mathrm{w}$ threshold where the packing of the droplets changes the emulsion's microscopic structure giving rise to Van der Waals forces-driven phenomena. Finding this threshold allowed expanding the concentration ranges of previously reported models. The main expectation is that these results will aid researchers and process/product designers to optimize their work in different industrial applications.
\end{abstract}

\section{Introduction}

Over the past decade, the design of chemical products has been revolutionized as new methods for modeling and predicting the final properties of a product using informatics tools have been proposed [1-4]. Recent research on product design has led to a shift in the focus from a business-to-business (B2B) to a business-to-consumer (B2C), bringing more attention to the quality of the final product, the ingredients used for its manufacture, and the final structure which impacts its performance [5-7].

With this in mind, multiple integrative approaches comprising marketing, management, finance and economics, product design and prototyping, process design, and manufacturing for the development of chemical-based products have been developed [8-11]. The increased computer capacity has allowed the integration of computer-aided tools to significantly improve product design. Most of these tools are based on optimization routines that rely on mathematical frameworks that model different stages of the product design (i.e., the manufacturing process, the final properties of the product, and the economic aspects of its commercialization) [12-14]. Thus, the importance of having a comprehensive knowledge of the relationship between the aforementioned stages is crucial.

A considerable number of studies have been conducted to consolidate mathematical models of emulsions and informatics tools to achieve a better product design. Among these studies, some propose general guidelines to design certain types of products [15-17], whilst others focus on 
specific applications [18-21]. Regardless of the type of application, the common factor among the cited studies remains understanding the basic phenomena and mathematical formulas that allow modeling the interactions between the properties, the formulation, and the manufacturing process through which a product is made.

One of the most common types of chemical goods in the B2C market is emulsion-based products [22]. Emulsions are a type of the colloidal system made of two immiscible liquid phases. The mixture of these phases is highly unstable from a thermodynamic and kinetic point of view, and therefore, the dispersion of droplets of one of the phases into the other requires an input of energy (provided by shear through a mixing device) and a surface-active species for stabilization. Emulsions are used in a wide range of industrial applications, such as pharmaceutics, food additives, petrochemicals, and cosmetics [19].

A multiscale approach for product design is based on the premise that the final properties of a product can be related to the process through which it was manufactured $[2,23]$. Therefore, any level of a property at the macroscopic, microscopic, or molecular scale can be tailored by modifying the manufacturing process at an industrial level. It follows that three different types of variables can be linked: the first ones are the requirements of a product, such as its composition or formulation; the second are process variables related to the manufacturing of the emulsion, such as the energy that was incorporated through the emulsification process; and third, the final properties of the emulsion which can be conceived from three scales: first, the macroscopic properties represented by the rheology and the energy required to emulsify; second, the microscopic properties as the structure and size distribution of the droplets, and third, the molecular properties represented by droplet-droplet and surfactant-surfactant interactions at the liquid-liquid interface $[24,25]$.

Historically, mathematical links between these properties have been described by numerous authors [26, 27]. However, most of the studies were focused on specific products, yielding mathematical relationships that are only applicable to the same or very similar emulsion systems. Some examples are mentioned as follows.

Regarding the petroleum industry, Azodi and Solaimany Nazar [28] proposed a model that links the surfactant concentration, the oil concentration, the salinity, the mixing speed, and the temperature to the viscosity of heavy crude oil in water emulsions. A similar study by Gingras et al. [29] concluded that for bitumen-in-water emulsions, the mean droplet size decreases if the agitation speed increases or if the bitumen concentration decreases.

In the food industry, Di Bari et al. [30] pointed out that water-in-cocoa oil emulsions shear during the emulsification process has an inversely proportional and exponential effect on the mean diameter of the droplets. The relationship of the droplet size and the applied shear is attributed to the breakdown of bigger droplets and the increased stability due to the dispersion of smaller droplets. Furthermore, Sato et al. [31] concluded that for emulsions stabilized by sodium caseinate at low concentrations of the dispersed phase, the pseudoplasticity of the emulsions depended on the higher structuration of their microscopic network. A link between the final product properties with process variables, such as the laccase treatment and the $\mathrm{pH}$, was proposed.

To present more general relationships that can be extrapolated to products across multiple industrial applications, some studies have been focused on a basic emulsion model, yielding relationships that would be valid for all the products that are based on the same colloidal system regardless of the nature of the components. Some of these studies are mentioned as follows.

Baldyga et al. [32] by means of computer fluid dynamics (CFD) concluded that the stresses in turbulent flow during the emulsification process were linked to the final droplet size. The way these stresses can be controlled is by adjusting the rotor speed of the impeller, that is, by varying the energy incorporated into the system, which means that the tip velocity is not being considered as a crucial parameter.

Masalova and Malkin [33] reported that in highly concentrated water-in-oil (W/O) emulsions with the content of the dispersed phase up to $96 \% w / w$, the plateau value of the elastic modulus $\left(G^{\prime}\right)$ is directly proportional to the average droplet diameter $(D)$ and that it follows the relationship of the following equation:

$$
G^{\prime} \propto D_{[4,3]}^{-2}
$$

This relationship was also found by Alvarez et al. [34] using water-in-mineral oil emulsions at concentrations higher than $90 \% w / w$ and by Paruta-Tuarez et al. [35] in W/ O emulsions within a concentration range between $89 \% \mathrm{w} / \mathrm{w}$ and $97 \% w / w$. To this date, these relationships are still used in different product design studies [36].

Despite this, most of the available studies focused on highly concentrated emulsions $(\phi>85 \% w / w)$ which means that the results are not necessarily valid for intermediate $(60<\phi<85 \% w / w)$, lower $(5<\phi<60 \% w / w)$, or dilute $(\phi<5 \% w / w)$ concentration ranges [37, 38]. Recent developments in the food and biomedical industries have benefited from low-concentration emulsions leveraging the low interdroplet interactions and stability [39-41]. For these applications, the previously described relationships are inaccurate and might lead to the incorrect design of products. To address this issue, in this study, a complete and transversal work is proposed to cover a wider range of concentrations and to extend the validity of the different relationships.

The first part of this article is dedicated to the study of a relationship between the energy incorporated through the emulsification process $\left(E_{v}\right)$, the De Brouckere mean droplet diameter $\left(D_{[4,3]}\right)$, and the elastic modulus $\left(G^{\prime}\right)$ in the linear viscoelastic region (LVE) of mineral oil-in-water emulsions $(\mathrm{O} / \mathrm{W})$ in a concentration range between $10 \%$ and $90 \% \mathrm{w} / \mathrm{w}$. The goal of this part is to expand the concentration range studied by Pradilla et al. [23] who established relationships between these variables using $\mathrm{O} / \mathrm{W}$ emulsions in a concentration range between $80 \%$ and $90 \% w / w$. In their study, it was found that the elastic modulus and the energy are correlated by equations (2) and (3) when the concentration is between $80 \%-85 \% w / w$ and $85 \%-90 \% w / w$, respectively. 


$$
\begin{aligned}
& G^{\prime} \propto E_{v}^{4}, \\
& G^{\prime} \propto E_{v}^{3} .
\end{aligned}
$$

For the same concentration ranges, a relationship between the mean diameter and the incorporated energy was proposed as shown in equations (4) and (5). If the elastic modulus is linked to the mean droplet diameter using these equations, a relationship following an approximation to equation (1) can be deducted.

$$
\begin{aligned}
& D_{[4,3]} \propto E_{v}^{-2.5}, \\
& D_{[4,3]} \propto E_{v}^{-1.5} .
\end{aligned}
$$

The second part of this work focuses on the study of the relationship between the flow behavior index and the dispersed phase packing at different concentrations considering the different microscopic configurations, a result of different kinetic phenomena. It is known that emulsions behave as shear-thinning fluids exhibiting a viscosity reduction when the shear rate increases [42]. A way to characterize this viscosity decrease is by using the flow behavior index $(n)$ approximating the behavior of the viscosity to a power-law model. Once again, in various studies, the flow behavior index for specific systems has been measured [43-45], but a general relationship between this variable and the incorporated energy has not been reported to the best of our knowledge.

\section{Materials and Methods}

2.1. Materials. In order to prepare the emulsions, mineral oil (USP-grade, viscosity $22.78 \mathrm{cp}$, density $0.87 \mathrm{~g} / \mathrm{cm}^{3}$ ) and Milli-Q deionized water were used as the dispersed and continuous phase, respectively. The emulsions were stabilized using two nonionic surfactants provided by Croda $($ : Span $80^{\circledR}$ (sorbitan monooleate) HLB 4.3 and Tween $20^{\circledR}$ (polyoxyethylene (20) sorbitan monolaurate or polysorbate 20) HLB 16.7.

\subsection{Methods}

2.2.1. Emulsion Manufacture. The emulsification process to prepare all the emulsions of this work is divided into three steps.

(1) Continuous and Dispersed Phase Prehomogenization. Tween $20^{\circledR}$ was mixed with water and Span $80^{\circledR}$ was mixed with the oil with a propeller-type impeller at 300 RPM using a mixing device (Lightnin Labmaster, Germany). The total amount of surfactant was $4 \% w / w$, and the ratio of the two surfactants was set to achieve a total HLB of 13. Transmission and backscattering measurements (Formulaction Turbiscan Lab Analyzer, France) at different total surfactant concentrations and HLB values were performed to follow possible instability phenomena (creaming, sedimentation, flocculation, Ostwald ripening, coalescence, or phase separation) during the experimental window of the emulsions of this work. As a result, the aforementioned parameters allowed maximum stability within the experimental window avoiding phase separation or surfactant agglomeration that could negatively influence droplet size measurements. Within these parameters, $0 \%$ of phase separation was observed.

(2) Incorporation of the Dispersed Phase. The oil phase was incorporated into the aqueous phase at a rate of $0.25 \mathrm{~mL} / \mathrm{s}$ using a peristaltic pump (Fischer Scientifics, USA). A propeller-type impeller was used with a tip speed of $1.7 \mathrm{~m} / \mathrm{s}$ in a system where the impeller-to-tank diameter ratio was 0.78 . To avoid undesired temperature effects, the process was performed at $40^{\circ} \mathrm{C}$.

(3) Homogenization. After incorporation of the dispersed phase, emulsions were homogenized for $10 \mathrm{~min}$ at the same conditions of the incorporation step.

Emulsions were prepared within a concentration range of $10-90 \% w / w$ with concentration increments of $10 \% w / w$. Unless otherwise specified, reproducibility was ensured by performing all measurements in triplicate. Results correspond to the average of all tests. The standard deviation of the measurements is shown for all figures, and its inverse was used as weights for all the equations fits. All the emulsions were characterized $5 \mathrm{~min}$ after the homogenization step.

2.2.2. Emulsion Characterization. The energy incorporated through the emulsification process was determined by numerical integration of the torque vs. time data normalized by the final volume of the emulsion.

The rheological behavior of the emulsions was measured using a hybrid rheometer (DHR-1, TA Instruments, UK) equipped with a concentric cylinder geometry at a constant gap of $5920 \mu \mathrm{m}$ and a temperature of $40^{\circ} \mathrm{C}$, whenever the oil concentration was lower or equal to $70 \% \mathrm{w} / \mathrm{w}$ and a $20 \mathrm{~mm}$ parallel plate geometry at a constant gap of $1000 \mu \mathrm{m}$ and a temperature of $40^{\circ} \mathrm{C}$, whenever the oil concentration was higher than $70 \% w / w$. Due to the transition of the emulsion behavior from liquid-like to solid-like, a change in the rheometer geometry was needed to guarantee the best experimental measurements given the emulsion properties (avoiding both the partial sample loss of liquid-like emulsions in the parallel plate geometry and Taylor vortices caused by the inertia of solid-like emulsions in the concentric cylinder geometry).

Flow tests were performed at a shear rate range of $1-100 \mathrm{~s}^{-1}$ and a constant temperature of $40^{\circ} \mathrm{C}$. The flow behavior index was calculated through an exponential regression of the viscosity vs. shear rate data. The elastic modulus $\left(G^{\prime}\right)$ was obtained through angular frequency oscillatory tests. The tests were performed in two steps: the first one was a frequency sweep between 0.1 and $300 \mathrm{rad} / \mathrm{s}$ at a constant oscillatory stress of $1 \mathrm{~Pa}$ and the second one a stress sweep between 0.1 and $300 \mathrm{~Pa}$ at a constant frequency in the plateau zone of the previous step. These two steps allowed the obtention of a stressand frequency- independent plateau value of the elastic modulus. 
The droplet size distribution and characteristic diameters were obtained using a MasterSizer 3000 (Malvern Instruments) using the laser diffraction technique, and the results were interpreted through the Mie theory. The mean diameter that was used in the study is the De Brouckere diameter also known as the $\mathrm{D}_{[4,3]}$ diameter.

\section{Results and Discussion}

3.1. Part I: Multiscale Approach. The multiscale approach is implemented by linking process variables (i.e., incorporated energy) with product properties (at the microscopic and macroscopic scales), across a wide range of possible product requirements (dispersed phase concentration and low shear rate viscosity).

The rheological behavior of the emulsions of this work is such that the elastic response is only measurable at concentrations of the dispersed phase greater than or equal to $60 \% w / w$ because a plateau region is observed in the first frequency sweep as shown in Figure 1. This means that at lower concentrations, the response of the systems is dominated by viscous dissipation and a frequency-independent response is not observed. Therefore, no elastic modulus values could be obtained in this area. The plateau region of the elastic modulus was associated with the rubbery behavior of different colloids [46, 47]. Across all the emulsions of this study, the values of the elastic modulus whenever a plateau region was found were always higher than the viscous modulus at the same frequencies, which yields information on the elastic nature of the system (the complete information of the viscous and elastic moduli is given in Figures S1-S5).

In order to explain why this behavior is only seen in emulsions with concentrations of the dispersed phase greater than $60 \% w / w$, optical microphotographs (Figure 2) were analyzed. It is seen that the concentration of $60 \% \mathrm{w} / \mathrm{w}$ marks a threshold; at immediate lower concentrations (i.e., $50 \% w / w)$, a higher interdroplet distance is observed. It can be inferred that the distance between droplets will increase at lower concentrations hindering the droplet-droplet interactions. Experiments showed that in most cases, the interdroplet contact is frequent even if critical packing $(\approx 75 \% w / w)$ has not been reached.

It can be hypothesized that the elastic moduli values in this region are related to droplet-droplet interactions. This hypothesis agrees with the results shown by Derkach [48] who established that there is an increment in viscosity at concentrations higher than $60 \% \mathrm{w} / \mathrm{w}$ due to the interactions between droplets (e.g., even inducing droplet deformation). Further than the already reported increment in the emulsion viscosity, these interactions give the system more elasticity because the droplets tend to align in a well-structured network with the ability to recover their shape once stress is applied, which is by definition an elastic-solid behavior $[42,49]$. Previous studies ascribed the increase of the elastic modulus beyond the critical packing concentration to the change in droplet geometry into complex amorphous polyhedrons that increases the contact between surfaces and their interactions as a consequence [50]. Our hypothesis also agrees with these studies but sets the precedent that the

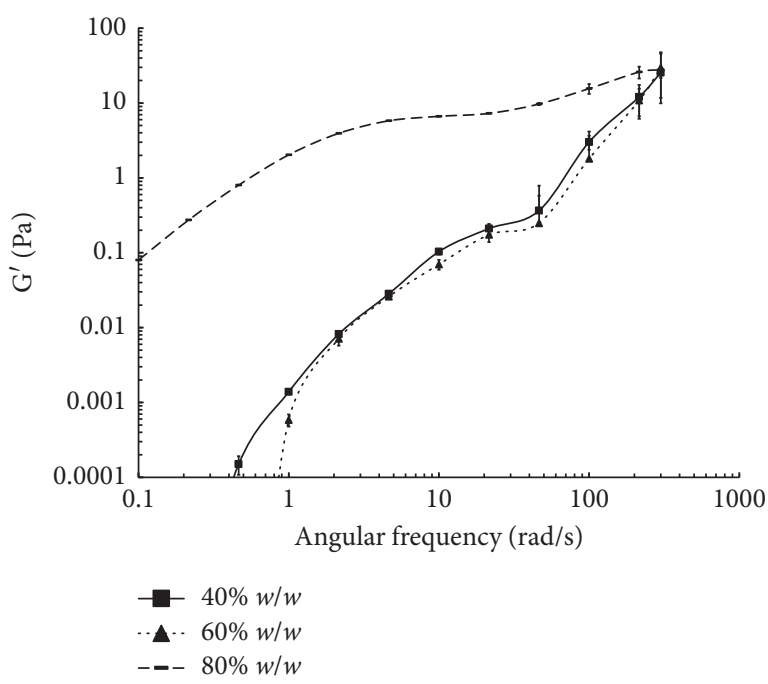

FIGURE 1: Frequency sweep of $40 \% w / w, 60 \% w / w$, and $80 \% w / w \mathrm{O} /$ $\mathrm{W}$ emulsions. A plateau or rubbery behavior zone is not present at concentrations lower than $60 \% \mathrm{w} / \mathrm{w}$ and is enhanced beyond the threshold. This is indicative of the difference in the "liquid-like" nature of low-concentration emulsions as opposed to the "solidlike" nature of highly concentrated systems.

critical packing is not a necessary condition to see an increase in the elastic modulus. We acknowledge that the change in shape from spheres to polyhedrons will increase the contact area between droplets (thus increasing interactions), enhancing, even more, the increase in the elastic modulus.

The increase of the elasticity in the system will significantly affect the process variables. In order to maintain the tip speed of the impeller during the emulsification step as the concentration increases, a greater strain will be required to the impeller to countervail the increase of the elasticity and viscosity of the system. From a quantifiable perspective, the greater strain will result in an increase in the energy used in the process. The energy incorporated through the emulsification step as a function of the emulsion concentration is shown in Figure 3. At concentrations lower than $60 \% w / w$, where the droplet interactions are minimal (as previously stated), the energy incorporated increases linearly. When the $60 \% \mathrm{w} / \mathrm{w}$ concentration threshold is reached and interdroplet interactions start to dominate due to their proximity, the elasticity of the system requires more energy and the energy requirement becomes exponential.

The exponential increment in the energy can also be analyzed from a microscopic perspective. At higher concentrations, additional energy is demanded from the system to break the droplets and reduce their size in order to achieve an increased packing degree [51]. It is known that the relationship between the droplet mean diameter and the energy incorporated is exponential and the resulting exponent depends on the emulsification process $[47,52]$. The exponential relationship between the mean diameter and the incorporated energy can be fit and approximated from Figure 4, giving rise to equation (6). This equation does not directly agree with the previous results found by Pradilla 


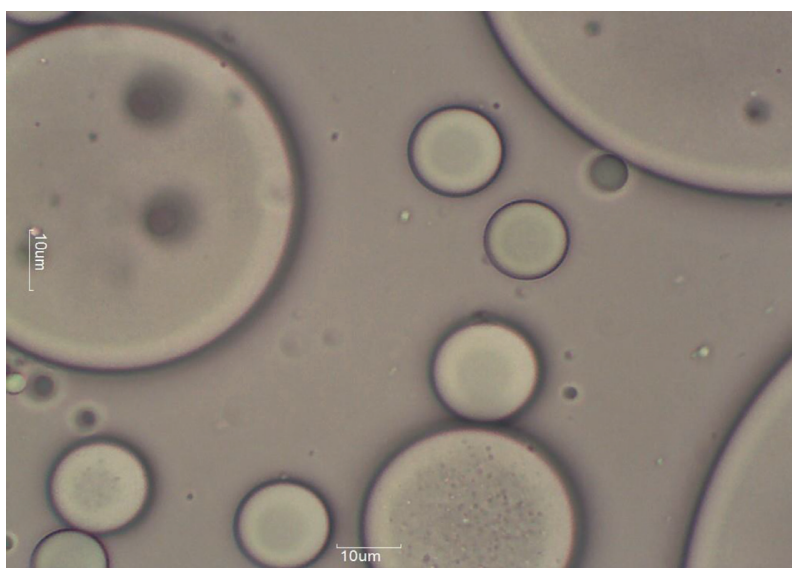

(a)

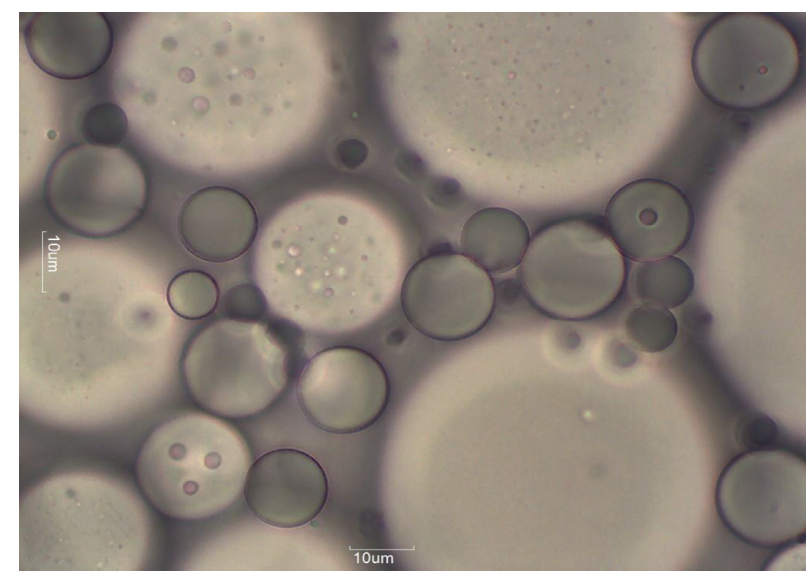

(b)

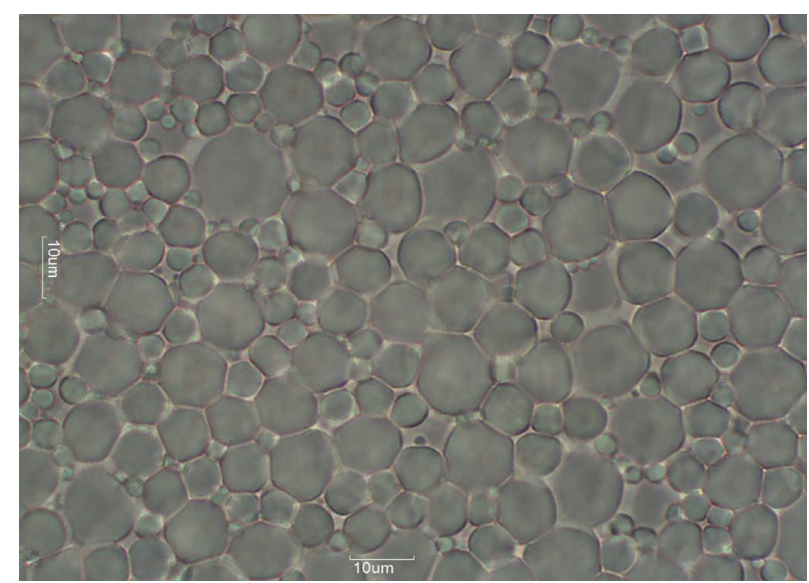

(c)

Figure 2: Optical microphotograph of $\mathrm{O} / \mathrm{W}$ emulsions in the $60 \% w / w$ proximity window. As the concentration reaches the $60 \% w / w$ threshold, the average distance between droplets is reduced and adjacent droplets are more common. (a) 50\%w/w. (b) 60\%w/w. (c) $80 \% w / w$. Scale $10 \mu \mathrm{m}$.

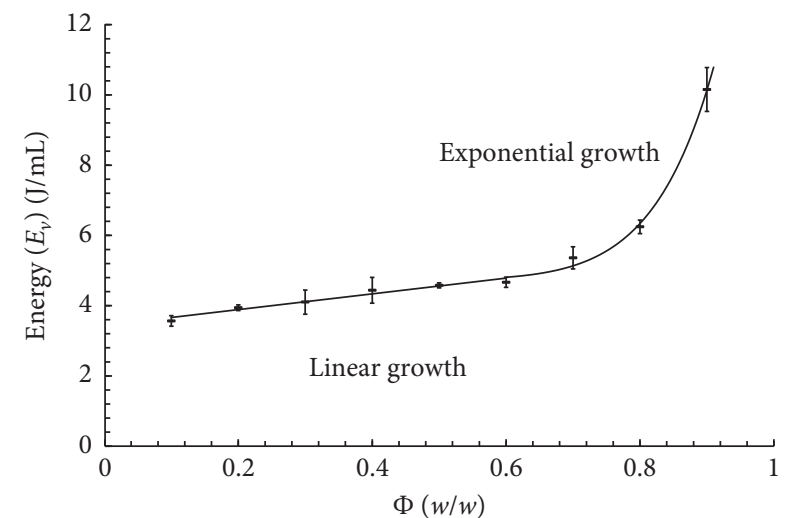

FIGURE 3: Energy incorporated per volume as a function of the concentration of the dispersed phase for $\mathrm{O} / \mathrm{W}$ emulsions within a concentration range of $10-90 \% w / w$. Two sections can be found: a linear growth below the $60 \% \mathrm{w} / \mathrm{w}$ threshold and an exponential growth afterwards. The change in the growth can be explained by the decrease in the interdroplet distance which enhances the interactions between droplets.

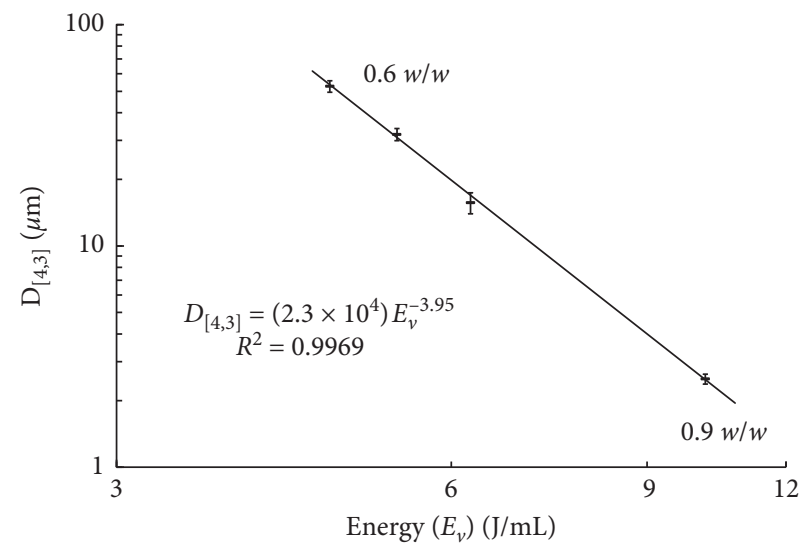

Figure 4: Droplet mean diameter $\mathrm{D}_{[4,3]} \mu \mathrm{m}$ (in log scale) as a function of the energy incorporated per volume (in log scale) for $\mathrm{O} /$ $\mathrm{W}$ emulsions within a concentration range of $60 \%-90 \% \mathrm{w} / \mathrm{w}$. The exponential relationship between the droplet size and the incorporated energy sheds light on additional reasons why the energy requirement increases with concentration. 
et al. [23] as shown in equations (4) and (5). We hypothesize that the difference is due to the wide concentration range that was used in our analysis; in the case of previous studies, the exponents were highly dependent on the concentration and a small difference in the ranges (i.e., $80 \%-85 \% w / w$ and $85-90 \% w / w)$ which led to numerical variations. As the concentration ranges distance from the maximum concentration, the proportionality exponent decreases until it reaches the value of the more general exponent that was found in this study.

$$
D_{[4,3]} \propto E_{v}^{-4} .
$$

The relationship between the macroscopic rheological properties of a system and the microscopic response has been widely studied. In this case, the elastic modulus was related to the droplet mean diameter as shown in Figure 5. It was found that the elastic modulus is proportional to the inverse of the mean diameter (squared), which agrees with the results of Alvarez et al. [34], Masalova et al. [25], ParutaTuarez et al. [35], and Pradilla et al. [23] as shown in equation (1). However, it is important to mention that these studies were conducted on highly concentrated emulsions where the concentration of the dispersed phase was higher than $80 \% \mathrm{w} / \mathrm{w}$. Our results expand the concentration range where the previously proposed expression is valid to concentrations higher than $60 \% \mathrm{w} / \mathrm{w}$.

From a molecular standpoint, the increase of the elasticity of the system is attributed to Van der Waals attractive forces caused by the increase of the interfacial interactions when the droplets form structured networks $[35,52]$. As discussed before, starting from a $60 \% \mathrm{w} / \mathrm{w}$ concentration, the droplets start forming a network with a large interfacial area. As the concentration increases, the degree of packing also increases, and droplet deformation becomes significant. Consequently, diverse polyhedral structures that allow larger contact areas between droplets are formed. As the tip speed of the impeller is maintained constant, the required strain to break the structures will increase exponentially, making the energy consumption increase at the same rate. This hypothesis agrees with the results found by Franco et al. [53] who report an exponential increase in the Metzner-Otto constant (i.e., the ratio between the effective shear rate and the stirrer speed) with increasing rheological parameters).

\subsection{Part II: Flow Behavior Index and Energy Incorporated.} The second part of this work is dedicated to establishing a link between the flow behavior index and the energy incorporated through the emulsification process. Several studies have been dedicated to developing mathematical relationships between the viscosity of an emulsion and other properties [54]. However, recent studies have also showed the importance of the flow behavior index in the product design [55].

Figure 6 shows the flow behavior index as a function of the energy incorporated. From this plot, it can be seen that at concentrations between $10 \% w / w$ and $20 \% w / w$, the emulsions show a Newtonian behavior. This phenomenon can be explained by the fact that there is not enough dispersed

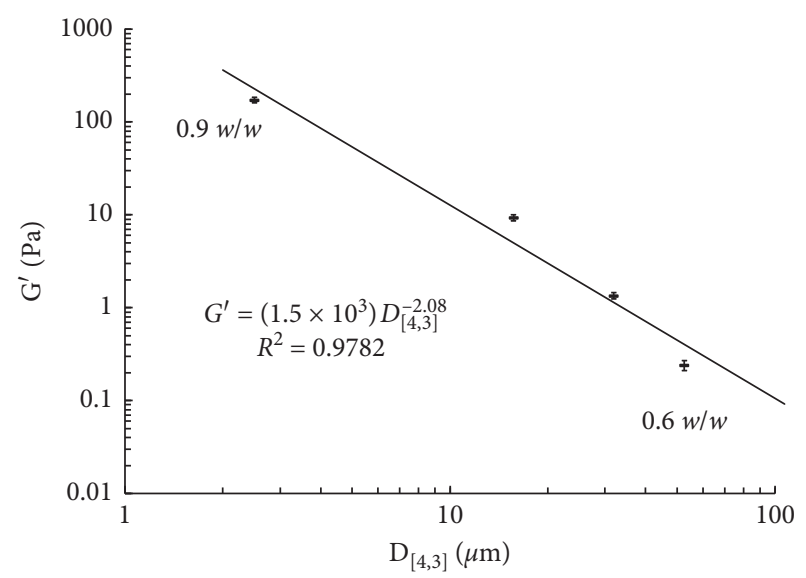

Figure 5: Stress- and frequency- independent elastic modulus $\left(G^{\prime}\right)$ as a function of the droplet mean diameter $\mathrm{D}_{[4,3]} \mu \mathrm{m}$ for $\mathrm{O} / \mathrm{W}$ emulsions within a concentration range of $60-90 \% w / w$ (the concentration values in descending order are $90,80,70$, and $60 \% w / w)$. The exponential fit matches what has been found in previous studies for highly concentrated systems. We show that the relationships are still valid to lower concentrations having $60 \% \mathrm{w} / \mathrm{w}$ as a lower bound.

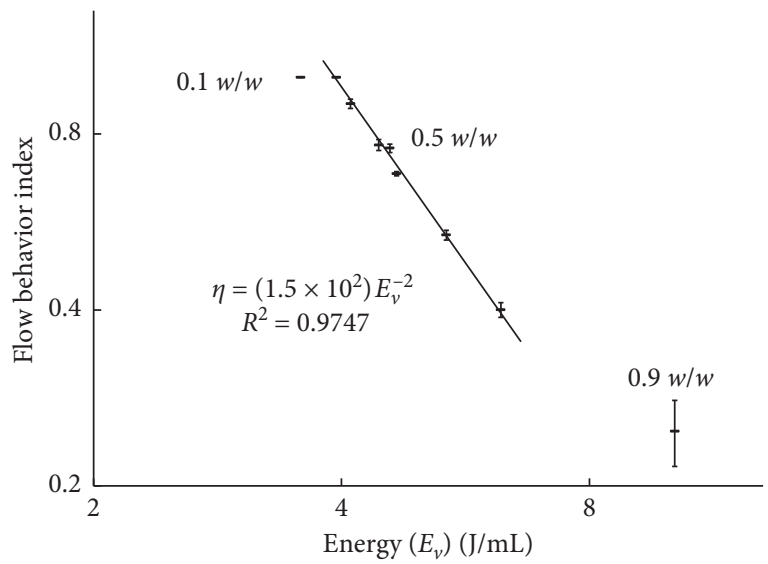

Figure 6: Flow behavior index (in log scale) as a function of the incorporated energy per volume (in log scale) for $\mathrm{O} / \mathrm{W}$ emulsions within a concentration range between $10 \% \mathrm{w} / \mathrm{w}$ and $90 \% \mathrm{w} / \mathrm{w}$ (the concentration values increase in $10 \% \mathrm{w} / \mathrm{w}$ intervals). Excluding the points where the dispersed phase concentration is too low to have an impact on the rheological behavior of the system or too high to let the system rearrange after a strain, a novel, and concentrationindependent relationship is proposed seen in the regression of the data.

phase to affect the viscosity of the system and so the emulsion takes on the properties of the continuous phase, in this case, water [56]. As the concentration increases, the flow behavior index decreases, and so the emulsions exhibit a more evident shear-thinning behavior.

The reduction of the flow behavior index appears to be divided into two different semiexponential zones: the first one determined by a concentration range between $20 \% \mathrm{w} / \mathrm{w}$ and $50 \% w / w$ and a second one within a concentration range between $50 \% w / w$ and $90 \% w / w$. These two zones will be described separately. 
As it was stated in the previous section and seen in Figure 2, in emulsions at lower concentrations, the distance between droplets is high, and the interdroplet interaction is low. Though some of the droplets tend to flocculate, a structured network is not formed, and the sphere-like shape of the droplets is not compromised. On the other hand, as the concentration increases and once the $60 \% \mathrm{w} / \mathrm{w}$ concentration threshold is reached, a structured network is formed. Beyond the packing limit, the droplets depart from their spherical shape to a polyhedral shape and are randomly arranged to form ordered structures [57, 58]. Rheological implications of droplet rearrangement have been previously discussed [59].

In the first zone, flocculation dominates, and if high shear is applied to the system, the emulsion will receive enough energy to break down the flocculated network. When flocs are formed, part of the continuous phase will be trapped between the droplets reducing the available space to move [60]. Flocs are continuously formed and destroyed, and the reduction of the viscosity is associated with the high destruction of the previously formed flocs [61]. When flocs are broken, the continuous phase is released allowing the system to flow without difficulty [62].

In the second zone, the formation of a structured network of droplets is the predominant phenomenon. The viscosity increases as a result of the increasing interactions between droplets at the liquid-liquid interface when the networks are formed $[48,49]$; in this case, higher shear will rearrange the structures in more ordered networks that allow the continuous phase to flow, thus obtaining a reduction in the viscosity.

A regression was applied to the data yielding the trend shown in Figure 6. A relationship between the flow behavior index and the energy incorporated through the emulsification process can be established and described by the following equation.

$$
\eta \propto E_{v}^{-2}
$$

The deviation from this relationship for emulsions at $10 \% w / w$ can be explained by considering that there is not enough dispersed phase to change the behavior of the system, letting the system behave solely as the continuous phase. The deviation at $90 \% w / w$ is related to the amount and degree of packing of the droplets that makes the system harder to rearrange [63].

Based on this relationship, the non-Newtonian behavior of a product based on an oil-in-water emulsion can be avoided or enhanced by varying the energy incorporated. This is an important remark that departs from the typical formulation-based product design that can be helpful for future product optimization.

\section{Conclusions}

In this work, a multiscale approach for studying the behavior of emulsion-based products was conducted. Mathematical relationships that link the incorporated energy through the emulsification process, the average droplet diameter, and the rheological behavior (in the form of elastic modulus and the flow behavior index) of $\mathrm{O} / \mathrm{W}$ emulsions were proposed.

The energy consumption as a function of the concentration of the dispersed phase during the manufacturing process was divided into a low-concentration region with linear behavior and a higher concentration region with an exponential behavior. The transition is seen at the point where the critical packing of the droplets drives an increase in the interdroplet Van der Waals interactions.

Our experiments show that the mean diameter is inversely proportional to the incorporated energy to the fourth exponent $\left(D_{[4,3]} \propto E^{-4}\right)$ and that the elastic modulus is inversely proportional to the squared mean diameter $\left(G^{\prime} \propto D_{[4,3]}^{-2}\right)$. These relationships are valid for all the concentrations where the elastic modulus is predominant over the loss modulus in oscillatory tests, improving previous concentration-specific relationships.

Finally, for a wide range of emulsion concentrations $(20-80 \% w / w)$, an inverse and quadratic relationship between the flow behavior index and the incorporated energy $\left(\eta \propto E_{v}^{-2}\right)$ was proposed.

The proposed equations can be directly applied to optimization models in the framework of product design. Recent studies have explored cases where the economic expenditures of emulsion systems are optimized considering the energy input as it contributes directly to production costs $[4,10,64]$. Our work adds equations that model the energy input as a function of the final properties that are usually set by the nature of the product (i.e., concentration of disperse phase and elastic modulus). Other studies have explored the effect of the flow behavior index on biomedical and food emulsions leading to restrictions in certain products [65-68]. Our results can be used to aid in tailoring this important rheologic property modifying the energy input to meet the specific requirements of the products. Furthermore, our explanations for the mechanisms behind the proposed relationships can give a broader picture for product design that can be considered for more complex analysis such as CFD-based product optimization.

\section{Data Availability}

The data used to support the findings of this study are available from the corresponding author upon request.

\section{Conflicts of Interest}

The authors declare that they have no conflicts of interest.

\section{Supplementary Materials}

The following information is available as supplementary material. Figure S1. The elastic and loss moduli for $\mathrm{O} / \mathrm{W}$ emulsions at a concentration of the dispersed phase of $50 \% \mathrm{w} / \mathrm{w}$ showing the liquid-like nature of the system. Figure S2. The elastic and loss moduli for O/W emulsions at a concentration of the dispersed phase of $60 \% \mathrm{w} / \mathrm{w}$ showing the transition to solid-like behavior. Figure S3. The elastic and loss moduli for $\mathrm{O} / \mathrm{W}$ emulsions at a concentration of the 
dispersed phase of $70 \% w / w$ showing the development of a plateau region indicative of solid-like behavior. Figure S4. The elastic and loss moduli for $\mathrm{O} / \mathrm{W}$ emulsions at a concentration of the dispersed phase of $80 \% \mathrm{w} / \mathrm{w}$ showing a fully developed plateau region typical solid-like response dominance. Figure S5. The elastic and loss moduli for $\mathrm{O} / \mathrm{W}$ emulsions at a concentration of the dispersed phase of $90 \% w / w$ showing a consolidated and wide range plateau region typical solid-like response dominance. (Supplementary Materials)

\section{References}

[1] R. Gani and K. M. Ng, "Product design-molecules, devices, functional products, and formulated products," Computers \& Chemical Engineering, vol. 81, pp. 70-79, 2015.

[2] J. P. Gallo-Molina, N. Ratkovich, and Ó. Álvarez, "Multiscale analysis of water-in-oil emulsions: a computational fluid dynamics approach," Industrial \& Engineering Chemistry Research, vol. 56, no. 27, pp. 7757-7767, 2017.

[3] J. P. Gallo-Molina, N. Ratkovich, and O. Alvarez, "The application of computational fluid dynamics to the multiscale study of oil-in-water emulsions," Industrial \& Engineering Chemistry Research, vol. 57, no. 2, pp. 578-589, 2018.

[4] J. J. Torres, C. D. Tinjaca, O. A. Alvarez, and J. M. Gómez, "Optimization proposal for emulsions formulation considering a multiscale approach," Chemical Engineering Science, vol. 212, p. 115326, 2020.

[5] C. Wibowo and K. M. Ng, "Product-oriented process synthesis and development: creams and pastes," AIChE Journal, vol. 47, no. 12, pp. 2746-2767, 2001.

[6] M. Hill, "Product and process design for structured products," AIChE Journal, vol. 50, no. 8, pp. 1656-1661, 2004.

[7] S. Burnaz and P. Bilgin, "Consumer evaluations on brand extensions: B2B brands extended into B2C markets," Journal of Product \& Brand Management, vol. 20, no. 4, pp. 256-267, 2011.

[8] W. D. Seider and S. Widagdo, "Teaching chemical engineering product design," Current Opinion in Chemical Engineering, vol. 1, no. 4, pp. 472-475, 2012.

[9] F. P. Bernardo and P. M. Saraiva, "A conceptual model for chemical product design," AIChE Journal, vol. 61, no. 3, pp. 802-815, 2015.

[10] K. Y. Fung, K. M. Ng, L. Zhang, and R. Gani, "A grand model for chemical product design," Computers \& Chemical Engineering, vol. 91, pp. 15-27, 2016.

[11] N. M. P. Bocken, I. de Pauw, C. Bakker, and B. van der Grinten, "Product design and business model strategies for a circular economy," Journal of Industrial and Production Engineering, vol. 33, no. 5, pp. 308-320, 2016.

[12] C. Whitnack, A. Heller, M. T. Frow, S. Kerr, and M. J. Bagajewicz, "Financial risk management in the design of products under uncertainty," Computers \& Chemical Engineering, vol. 33, no. 5, pp. 1056-1066, 2009.

[13] M. Mattei, G. M. Kontogeorgis, and R. Gani, "A comprehensive framework for surfactant selection and design for emulsion based chemical product design," Fluid Phase Equilibria, vol. 362, pp. 288-299, 2014.

[14] F. P. Bernardo, "Integrated process and product design optimization," Computer Aided Chemical Engineering, vol. 39, pp. 347-372, 2016.
[15] G. Boothroyd, "Product design for manufacture and assembly," Computer-Aided Design, vol. 26, no. 7, pp. 505-520, 1994.

[16] E. Lutters, F. J. A. M. Van Houten, A. Bernard, E. Mermoz, and C. S. L. Schutte, "Tools and techniques for product design," CIRP Annals, vol. 63, no. 2, pp. 607-630, 2014.

[17] F. Ceschin and I. Gaziulusoy, "Evolution of design for sustainability: from product design to design for system innovations and transitions," Design Studies, vol. 47, pp. 118-163, 2016.

[18] J. Sjöblom, N. Aske, I. Harald Auflem et al., "Our current understanding of water-in-crude oil emulsions. recent characterization techniques and high pressure performance," Advances in Colloid and Interface Science, vol. 100-102, pp. 399-473, 2003.

[19] L. L. Schramm, Emulsions, Foams, and Suspensions: Fundamentals and Applications, Wiley, Hoboken, NJ, USA, 2006.

[20] G. Narsimhan and Z. Wang, "Guidelines for processing emulsion-based foods," in Food Emulsifiers and Their Applications, G. L. Hasenhuettl and R. W. Hartel, Eds., Springer, New York, NY, USA, pp. 349-394, 2008.

[21] D. T. Piorkowski and D. J. McClements, "Beverage emulsions: recent developments in formulation, production, and applications," Food Hydrocolloids, vol. 42, pp. 5-41, 2014.

[22] M. Mattei, G. M. Kontogeorgis, and R. Gani, "A systematic methodology for design of emulsion based chemical products," Computer Aided Chemical Engineering, vol. 31, pp. 220-224, 2012.

[23] D. Pradilla, W. Vargas, and O. Alvarez, "The application of a multi-scale approach to the manufacture of concentrated and highly concentrated emulsions," Chemical Engineering Research and Design, vol. 95, pp. 162-172, 2015.

[24] A. Y. Malkin, I. Masalova, P. Slatter, and K. Wilson, "Effect of droplet size on the rheological properties of highly-concentrated w/o emulsions," Rheologica Acta, vol. 43, no. 6, pp. 584-591, 2004.

[25] I. Masalova, R. Foudazi, and A. Y. Malkin, "The rheology of highly concentrated emulsions stabilized with different surfactants," Colloids and Surfaces A: Physicochemical and Engineering Aspects, vol. 375, no. 1-3, pp. 76-86, 2011.

[26] M. F. San Martin-González, A. Roach, and F. Harte, "Rheological properties of corn oil emulsions stabilized by commercial micellar casein and high pressure homogenization," LWT-Food Science and Technology, vol. 42, no. 1, pp. 307311, 2009.

[27] M. Ronald and F. P. Luis, "Asphalt emulsions formulation: state-of-the-art and dependency of formulation on emulsions properties," Construction and Building Materials, vol. 123, pp. 162-173, 2016.

[28] M. Azodi and A. R. Solaimany Nazar, "An experimental study on factors affecting the heavy crude oil in water emulsions viscosity," Journal of Petroleum Science and Engineering, vol. 106, pp. 1-8, 2013.

[29] J.-P. Gingras, P. A. Tanguy, S. Mariotti, and P. Chaverot, "Effect of process parameters on bitumen emulsions," Chemical Engineering and Processing: Process Intensification, vol. 44, no. 9, pp. 979-986, 2005.

[30] V. Di Bari, J. E. Norton, and I. T. Norton, "Effect of processing on the microstructural properties of water-in-cocoa butter emulsions," Journal of Food Engineering, vol. 122, no. 1, pp. 8-14, 2014.

[31] A. C. K. Sato, F. A. Perrechil, A. A. S. Costa, R. C. Santana, and R. L. Cunha, "Cross-linking proteins by laccase: effects on the droplet size and rheology of emulsions stabilized by sodium 
caseinate," Food Research International, vol. 75, pp. 244-251, 2015.

[32] J. Bałdyga, M. Jasińska, and A. J. Kowalski, "Effect of rheology of dense emulsions on the flow structure in agitated systems," Chemical Engineering Research and Design, vol. 108, pp. 3-12, 2016.

[33] I. Masalova and A. Y. Malkin, "Peculiarities of rheological properties and flow of highly concentrated emulsions: the role of concentration and droplet size," Colloid Journal, vol. 69, no. 2, pp. 185-197, 2007.

[34] O. A. Alvarez, L. Choplin, V. Sadtler et al., "Influence of semibatch emulsification process conditions on the physical characteristics of highly concentrated water-in-oil emulsions," Industrial \& Engineering Chemistry Research, vol. 49, no. 13, pp. 6042-6046, 2010.

[35] E. Paruta-Tuarez, P. Marchal, V. Sadtler, and L. Choplin, "Analysis of the princen and kiss equation to model the storage modulus of highly concentrated emulsions," Industrial \& Engineering Chemistry Research, vol. 50, no. 17, pp. 10359-10365, 2011.

[36] J. A. Arrieta-Escobar, F. P. Bernardo, A. Orjuela, M. Camargo, and L. Morel, "Incorporation of heuristic knowledge in the optimal design of formulated products: application to a cosmetic emulsion," Computers \& Chemical Engineering, vol. 122, pp. 265-274, 2019.

[37] W. D. Pandolfe, "Effect of dispersed and continuous phase viscosity on droplet size of emulsions generated by homogenization," Journal of Dispersion Science and Technology, vol. 2, no. 4, pp. 459-474, 1981.

[38] R. Pal, "Rheology of simple and multiple emulsions," Current Opinion in Colloid \& Interface Science, vol. 16, no. 1, pp. 41-60, 2011.

[39] H. Singh, "Aspects of milk-protein-stabilised emulsions," Food Hydrocolloids, vol. 25, no. 8, pp. 1938-1944, 2011.

[40] D. J. McClements, "Emulsion design to improve the delivery of functional lipophilic components," Annual Review of Food Science and Technology, vol. 1, no. 1, pp. 241-269, 2010.

[41] C. A. Fraker, A. J. Mendez, L. Inverardi, C. Ricordi, and C. L. Stabler, "Optimization of perfluoro nano-scale emulsions: the importance of particle size for enhanced oxygen transfer in biomedical applications," Colloids and Surfaces B: Biointerfaces, vol. 98, pp. 26-35, 2012.

[42] H. J. Butt, "Controlling the flow of suspensions," Materials Science, vol. 331, no. 6019, pp. 868-869, 2011.

[43] M. Maskan and F. Göğüş, "Effect of sugar on the rheological properties of sunflower oil-water emulsions," Journal of Food Engineering, vol. 43, no. 3, pp. 173-177, 2000.

[44] C. Sun, S. Gunasekaran, and M. P. Richards, "Effect of xanthan gum on physicochemical properties of whey protein isolate stabilized oil-in-water emulsions," Food Hydrocolloids, vol. 21, no. 4, pp. 555-564, 2007.

[45] H. Mohammadzadeh, A. Koocheki, R. Kadkhodaee, and S. M. A. Razavi, "Physical and flow properties of D-limonene-in-water emulsions stabilized with whey protein concentrate and wild sage (salvia macrosiphon) seed gum," Food Research International, vol. 53, no. 1, pp. 312-318, 2013.

[46] H. Niu, Y. Wang, X. Liu, Y. Wang, and Y. Li, "Determination of plateau moduli and entanglement molecular weights of ultra-high molecular weight isotactic polypropylene synthesized by ziegler-natta catalyst," Polymer Testing, vol. 60, pp. 260-265, 2017.

[47] T. F. Tadros, Emulsion Formation, Stability, and Rheology, Wiley, Hoboken, NJ, USA, 2013.
[48] S. R. Derkach, "Rheology of emulsions," Advances in Colloid and Interface Science, vol. 151, no. 1-2, pp. 1-23, 2009.

[49] N. G. Diftis, C. G. Biliaderis, and V. D. Kiosseoglou, "Rheological properties and stability of model salad dressing emulsions prepared with a dry-heated soybean protein isolate-dextran mixture," Food Hydrocolloids, vol. 19, no. 6, pp. 1025-1031, 2005.

[50] T. G. Mason, J. Bibette, and D. A. Weitz, "Elasticity of compressed emulsions," Physical Review Letters, vol. 75, no. 10, pp. 2051-2054, 1995.

[51] M. Kröger and J. Vermant, "The structure and rheology of complex fluids," Applied Rheology, vol. 10, no. 3, pp. 110-111, 2019.

[52] H. Schubert and H. Armbruster, "Principles of formation and stability of emulsions," International Journal of Chemical Engineering, vol. 32, no. 1, pp. 14-28, 1992.

[53] J. M. Franco, M. A. Delgado, C. Valencia, M. C. Sánchez, and C. Gallegos, "Mixing rheometry for studying the manufacture of lubricating greases," Chemical Engineering Science, vol. 60, no. 8-9, pp. 2409-2418, 2005.

[54] D. J. McClements, Food Emulsions: Principles, Practices, and Techniques, CRC Press, Boca Raton, FL, USA, Second edition, 2004.

[55] N. Dapueto, E. Troncoso, C. Mella, and R. N. Zúñiga, “The effect of denaturation degree of protein on the microstructure, rheology and physical stability of oil-in-water $(\mathrm{O} / \mathrm{W})$ emulsions stabilized by whey protein isolate," Journal of Food Engineering, vol. 263, pp. 253-261, 2019.

[56] N. Willenbacher and K. Georgieva, Product Design and Engineering: Formulation of Gels and Pastes, Wiley, Hoboken, NJ, USA, 2013.

[57] E. Dickinson, "Understanding food structures: the colloid science approach," in Food Structures, Digestion and Health, pp. 3-49, Elsevier, Amsterdam, Netherlands, 2014.

[58] M. Ochowiak, L. Broniarz-Press, and J. Rozanski, "Rheology and structure of emulsions and suspensions," Journal of Dispersion Science and Technology, vol. 33, no. 2, pp. 177-184, 2012.

[59] S. Cohen-Addad and R. Höhler, "Rheology of foams and highly concentrated emulsions," Current Opinion in Colloid \& Interface Science, vol. 19, no. 6, pp. 536-548, 2014.

[60] D. T. N. Chen, Q. Wen, P. A. Janmey, J. C. Crocker, and A. G. Yodh, "Rheology of soft materials," Annual Review of Condensed Matter Physics, vol. 1, no. 1, pp. 301-322, 2010.

[61] R. Pal, "Viscosity models for multiple emulsions," Food Hydrocolloids, vol. 22, no. 3, pp. 428-438, 2008.

[62] B. C. Tatar, G. Sumnu, and S. Sahin, "Rheology of emulsions," . In Advances in Food Rheology and its Applications, Elsevier, Amsterdam, Netherlands, pp. 437-457, 2017.

[63] Y. Zhu, H. Gao, W. Liu, L. Zou, and D. J. McClements, "A review of the rheological properties of dilute and concentrated food emulsions," Journal of Texture Studies, vol. 51, no. 1, pp. $45-55,2020$.

[64] M. Bagajewicz, S. Hill, A. Robben et al., "Product design in price-competitive markets: a case study of a skin moisturizing lotion," AIChE Journal, vol. 57, no. 1, pp. 160-177, 2011.

[65] A. R. Taherian, P. Fustier, and H. S. Ramaswamy, "Steady and dynamic shear rheological properties, and stability of nonflocculated and flocculated beverage cloud emulsions," International Journal of Food Properties, vol. 11, no. 1, pp. 24-43, 2008.

[66] V. Samavati, Z. Emam-Djomeh, M. A. Mohammadifar, M. Omid, and A. Mehdinia, "Application of rheological 
modeling in food emulsions," Iranian Journal of Chemistry and Chemical Engineering, vol. 31, no. 2, pp. 71-83, 2012.

[67] M. Sarker and X. B. Chen, "Modeling the flow behavior and flow rate of medium viscosity alginate for scaffold fabrication with a three-dimensional bioplotter," Journal of Manufacturing Science and Engineering Transactions of the ASME, vol. 139, no. 8, 2017.

[68] D. F. S. Petri, "Xanthan gum: a versatile biopolymer for biomedical and technological applications," Journal of Applied Polymer Science, vol. 132, no. 23, 2015. 